\title{
Active learning in intermediate optics through class tutorials and concept building laboratories
}

\section{Mark Masters, Timothy Grove}

Mark F. Masters, Timothy T. Grove, "Active learning in intermediate optics through class tutorials and concept building laboratories," Proc. SPIE 9666, 11th Education and Training in Optics and Photonics Conference, 96660K (5 June 2009); doi: 10.1117/12.2207952

Event: Eleventh International Topical Meeting on Education and Training in Optics and Photonics, 2009, St. Asaph, United Kingdom 


\title{
Active learning in intermediate optics through class tutorials and concept building laboratories Mark F. Masters ${ }^{a}$ and Timothy T. Grove ${ }^{b}$ Department of Physics \\ Indiana University - Purdue University Fort Wayne \\ Fort Wayne IN 46805, USA
}

\begin{abstract}
We have been modifying our intermediate optics class and laboratory with a focus on improving student learning through the use of active engagement. To facilitate this process we developed a two pronged solution. For the classroom we created a series of tutorials to help the students use the mathematics and techniques of derivations, apply these solutions to other problems, and develop a stronger conceptual foundation in intermediate optics class. In the optics laboratories we developed an approach that relies upon direct confrontation of misconceptions, predictions, collection of data to support or refute the predictions, reconciliation, discussion, and leading questions rather than a series of detailed, cookbook-like instructions as might be found in a traditional laboratory. Through the class and laboratory we build conceptual understanding in subjects like image formation by lenses and mirrors, ray optics, and ultimately elliptical polarization while fostering laboratory independence and helping students erect a new paradigm for learning.
\end{abstract}

Keywords: active learning, interactive engagement, optics tutorials, optics laboratory

\section{INTRODUCTION}

In 2006 we embarked on a complete revision of our optics curriculum which had not been updated for over twenty years. These revisions involved changing how we teach the intermediate optics lecture and the laboratory. One consideration in these modifications is that both the class and laboratory must be challenging for senior physics students who have significant laboratory and classroom experience while not being impossible for students who have just completed the introductory physics sequence.

The optics class and laboratory modifications follow in the wake of significant revisions of our introductory physics classes and laboratories. In the introductory classroom we make significant use of a teaching approach known as interactive engagement ${ }^{1}$ and peer teaching ${ }^{2,3}$ rather than simple lecturing. Given the measureable success of this approach at improving student understanding of the material ${ }^{1}$ and considerable change in student attitude to learning, we felt it would be appropriate to adopt interactive engagement for the lecture component of our intermediate optics class. However, there are difficulties with implementing interactive engagement at an intermediate level.

\section{WHAT ARE ACTIVE LEARNING AND INTERACTIVE ENGAGEMENT?}

Active learning and interactive engagement (IE) means different things to different people. Active learning is an educational technique that developed over time. It is an approach in which students are involved in, and responsible for their own learning process. Active learning uses various methods such as interactive lecture demonstrations ${ }^{4}$ and peer instruction ${ }^{2}$ to aid students in understanding the material under deliberation. Adoption of this approach tends to have wider implications on the students' learning methodology (knowledge acquisition vs. knowledge construction, answer making vs. sense making) than simply the material under consideration. Active learning requires the development of a habit of mind for both instructor and student. In

a $\quad$ masters@ipfw.edu, http:// users.ipfw.edu/masters

b_grovet@ipfw.edu 
comparison, a traditional lecture class is dominated by student passivity (sitting in class and listening to lecture) followed (hopefully) by independent active learning through homework (if properly designed) and text reading (if the students do it). In a traditional lecture class there is no need for students to mentally engage with the material while in class.

The term interactive engagement encompasses any and all of the variety of active learning approaches and venues, whether in the laboratory, lecture, studio ${ }^{5,6}$ or recitation format. ${ }^{\prime} I E$ classes can be characterized as a "minds-on, hands-on" approach; the students are mentally engaged with the material in the classroom and they must do the work in the class. The students actively discover the material and its intricacies in class. IE has been used extensively at the introductory physics level with great success in improving student understanding and retention of knowledge. ${ }^{7}$

An IE class is dependent upon the communication process. The instructor must be engaged in a conversation with the whole class (not with a single questioner) and as such discovers misconceptions held by the students. This enables the instructor to contrive situations to directly confront those misconceptions. The instructor's role is to set the scenario that the students investigate or consider; to determine the problem they have to solve and then allow the students to discuss the problem in small groups. Once a group consensus is formed, the instructor moderates a class discussion leading to a class consensus. The instructor may direct the conversation with particular questions, but the instructor is not the source of information. Rather, it is the class discussion that is the source of information.

\section{CHALLENGES}

In a traditional intermediate physics class, a significant amount of class time is devoted to the instructor showing the students derivations of key results. These results are then applied to a variety of physical situations. Similarly, in a traditional laboratory phenomena and classic investigations are generally demonstrated. Unfortunately, too many students do not or cannot connect the derivation to physics, they cannot follow the logical methodology applied through the derivation, and they are unable to apply laboratory results and techniques to other physical situations or investigations. For example, do students know under what situations the derivation results are valid? Can they apply the derivation techniques to different problems? Are the students learning effectively in the laboratory if they are only following directions to complete a demonstration?

We turned to IE in an attempt to improve learning in intermediate classes, but there are many issues. The lack of information available regarding student misconceptions of intermediate and advanced undergraduate topics makes this task more difficult than introductory courses. Furthermore, the nature of these courses simply makes it harder to implement IE. Some of the problems include: 1) the concepts become more challenging; 2) the mathematics is more challenging; 3 ) the students must become adept at interpreting the mathematics physically and relating the results to reality; 4) a traditional (and important) reliance on derivations; 5) the laboratory investigations become more technologically and conceptually complex; and 6) these classes build upon earlier classes and any misconceptions not allayed in those classes will cause difficulties in these.

Not to be dismayed, we have developed techniques to facilitate the use of $I E$ in the intermediate optics venue. Our solution has been to develop a series of tutorials for the lecture portion of the class and to completely redesign the laboratories to engage the students. Our basic philosophy is that the students must discover the physics. These tutorials and laboratories are available on our web page: http://users.ipfw.edu/masters/Optics ccli project.htm.

It is important for an instructor to recognize that adopting $I E$ in a class or laboratory may make progress through the materials more slow than in the case of traditional lecturing. As a result, the students will have a better mastery of the materials, improved learning skills, and better investigative skills. Regardless, we have to reduce some of the topics often considered in lecture and in laboratory due to time constraints. 


\section{THE LECTURE COMPONENT OF INTERMEDIATE OPTICS}

The intermediate optics course has two components: geometric optics and physical optics. These basic optics models then provide a strong foundation for more advanced courses. Additionally, we try to help the students become more sophisticated in their use of mathematics, to expand their ability to interpret mathematics physically and improve their conceptual understanding of light. Rather than lecturing or showing this material, we let the student discover the physics though tutorials.

\section{Course outline}

I. Nature and models of light

a. Basic ray model

b. Basic wave model (what is a wave)

c. Photonic model

II. Geometric optics
a. Point and extended sources
b. Ray-tracing
c. Images
d. Optical systems
e. Aberrations
f. Mathematical formalism

III. Physical optics

a. Mathematical wave formalism for light and Maxwell's equations

b. Polarization (polarizer concepts and matrix methods)

c. Interference and diffraction

The purpose of a tutorial is to help initiate the discussions. A tutorial is a document designed to lead a student to a solution and were inspired by Lillian McDermot's Tutorials in Introductory Physics ${ }^{8}$ and Alan van Heuvelen's Active Learning Problem Sets ${ }^{9}$. There are three classes of tutorials: introductory tutorials which should be a review and deal directly with misconceptions; guided derivation tutorials in which the students are helped through a derivation of some particular result; and application problems.

Typically, a class will use the sequence: 1 ) the students work in small groups on the tutorial while the instructor circulates and asks additional questions to various groups of students (based on where they encounter difficulties), 2) the class discusses the results, 3) resolution. Occasionally, a tutorial might be assigned for the students to complete at home for discussion in the subsequent class.

\section{Models of light}

In geometric optics we stress use and interpretation of ray diagrams. Often, the value of ray diagrams is not apparent to students since in class it is often rapidly replaced with equations in introductory physics. However, ray diagrams contain a wealth of information about images, locations where images can be seen, etc. that is not available through pure calculation. But to understand ray diagrams, the students must first construct an idea of what is meant by a ray of light. We ask questions to guide these discussions such as: "What is a light ray?" "What information about light does a single ray indicate? A collection of rays indicate?" "Using a ray diagram how would one indicate that one light beam has twice the power of another?" "How could you indicate irradiance using a ray diagram?"

Waves are a source of confusion for students. Typically students confound the amplitude of a wave with the spatial extent or physical size of the wave. Other commonly held beliefs of light-wave misconceptions: "light travels in waves," "light is composed of particles that are following a wave path," and "the static wave diagram represents the path followed by light." These misconceptions are also addressed through discussions.

Students are often confuse the amplitude of a wave in a diagram as indicating the spatial size of a wave. To address the spatial extent of a wave as relative to its spatial size, we consider a longitudinal wave. In figure 1 , the students are asked to plot the wave - which consists only of counting particles in a box. If we double 
the height of the boxes making the wave's spatial extent larger, do we change the amplitude of the wave? If we double the amplitude of the wave, what has to change with the number of particles in each box?
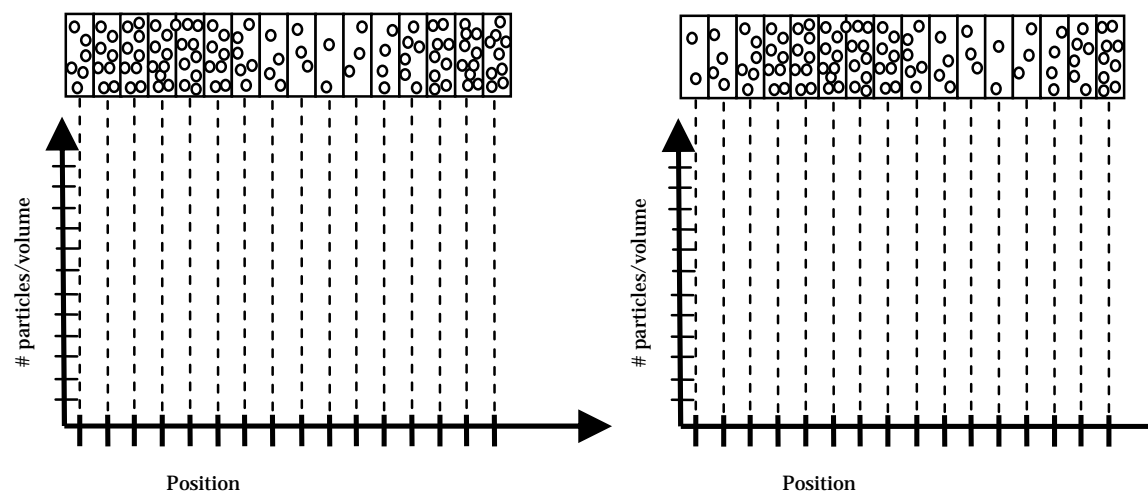

B
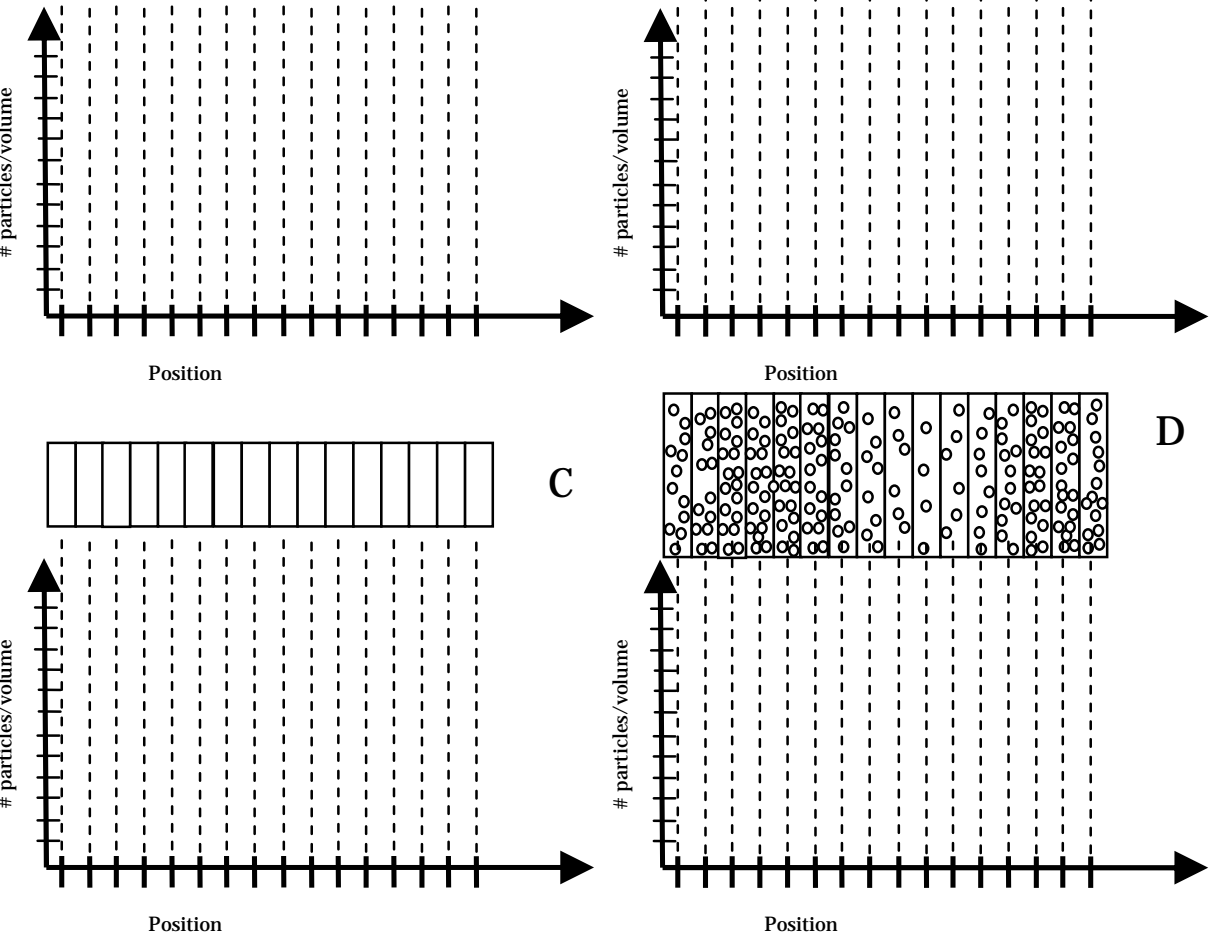

Figure 1 - Tutorial fragment to assist students in understanding the wave representation and also wavelength, frequency and how they are related. The divisions on each grid are every $5 \mathrm{~cm}$. The boxes are $5 \mathrm{~cm}$ wide by $10 \mathrm{~cm}$ high by $1 \mathrm{~cm}$ deep (into page). A) Plot the wave form on the supplied grid. B) The same wave but a short time later. Determine the speed, wavelength and frequency of the wave. C) Suppose you were to double the amplitude of the wave in A, make a graph and fill in the corresponding number of particles in each box. D) Suppose the volume of each box were doubled. What would change about the graphical representation? Would the physical dimensions (size or spatial extent) of the wave change?

The ray and wave models of light are commonly treated as independent. However, they share some common information which is worth exploring. Consider the question posed in Figure 2A and answered in Figure 2B.

A

Figures $2-$ A) The wave diagram of a beam of light passing through an optical element placed at the dashed line. Construct a ray diagram that contains some of the same information and determine, as specifically as possible, the type of optical element. B) the solution. 


\section{Ray tracing}

Once the idea of a ray diagram has been established the class works on imaging through ray tracing. Figure 3 is used to develop ray tracing formalism for a thin mirror. The students already have discussed ray diagrams and the law of reflection as found through Fermat's principle.

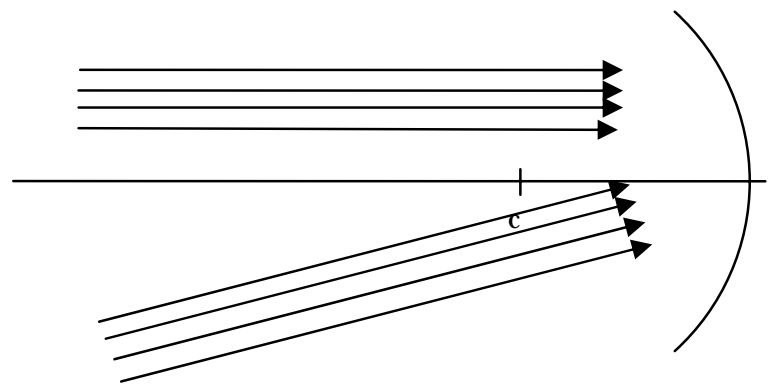

Figure 3 - Tutorial fragment showing the beginning of ray-tracing images for curved mirrors. The diagram showing the bundles of parallel rays serves several purposes. First, it forces the students to recognize that not all of these rays cross at the "focal point." Second it is a refresher that the bundle of parallel rays constitutes light from a distant point source. Third, distant point sources will have parallel bundles of rays that intercept the optic at different angles with respect to the optic axis.

Making the students work with ray tracing is important so that they develop an appreciation of what they can determine using this technique. In Figure 4 are two examples in which simple numerical calculations of image location and a lack of understanding of ray diagrams would make the questions unanswerable. (Figure $4 \mathrm{~B}$ based on techniques developed by Professor David P. Maloney ${ }^{10}$ )

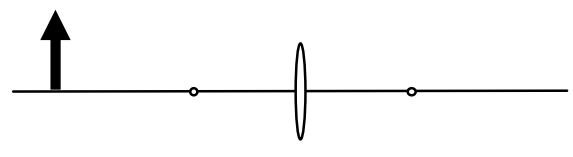

A

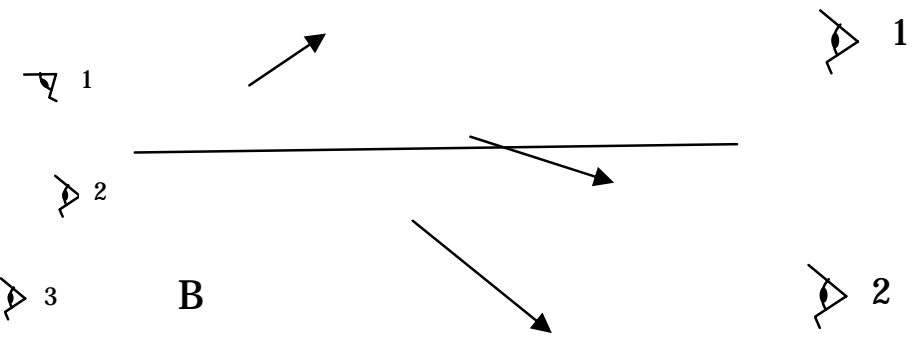

Figure 4 A) Describe what can each of the observers (1-4) see. B) The three ray segments shown are part of the different principle rays used for ray tracing. Based on this diagram, specify the type of lens, its focal length, image and object locations. Observer 1 can only see the bottom of the object. Observer 2 can see the whole object. Estimate the size of the lens.

\section{Derivations}

An important aspect of any intermediate class is the derivation. Rather than the instructor simply showing the students the derivations on the board and having the students copy it to their notes, we require the students work through the derivations in peer groups. For example, in Figure 5, the beginning of a tutorial intended to lead the students to apply the appropriate geometric construction and approximations to find the "thin mirror" equation and to determine the location of the "focal point" is shown. After the students have been given time to complete the tutorial, the class reconvenes and a discussion of the results of the derivation proceed, with the instructor asking questions about various details of the derivation. Other derivation tutorials have been developed that investigate spherical refracting surfaces, double refracting surfaces, aberrations, frustrated internal reflection, the eye, wave equations, interference and polarization. 


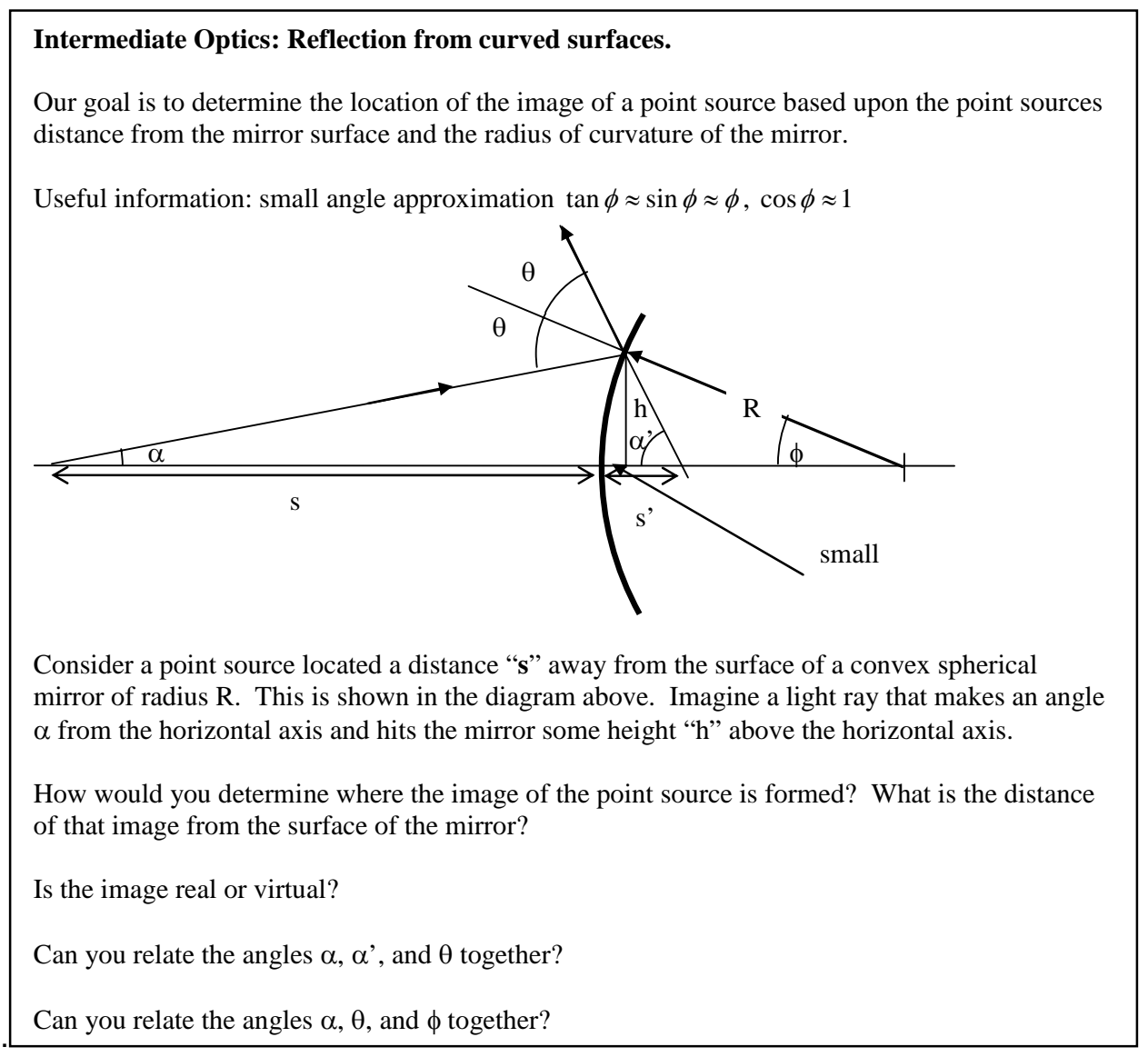

Figure 5 -Beginning of the derivation tutorial of the thin-mirror equation. Students have to work through the mathematics and then interpret the results.

\section{Application}

Application tutorials require the students to use the relations and concepts that they have discussed and derived. They are not asked to simply plug in numbers, but to synthesize various techniques, methods and concepts into a whole. Figure $4 \mathrm{~B}$ is an example of an application tutorial in which the students have to work with ray tracing. Another example is given in Figure 6.

\section{Tutorial results}

These tutorials serve several purposes. First, the instructor becomes apprised of the student's misconceptions and mathematical skills; information an instructor could not as easily obtain via lecturing. Secondly, the students leave the class with clear notes in the form of the completed tutorial rather than the haphazard collection of copied information. Furthermore, the critical discussion that takes place at the end of class forces the students to put the material in their own knowledge framework. We believe (and our observations support this) that after this class format, the majority of students have better retention of the optics concepts and develop better mathematical skills and seem to enjoy the collaborative nature of the classroom. 


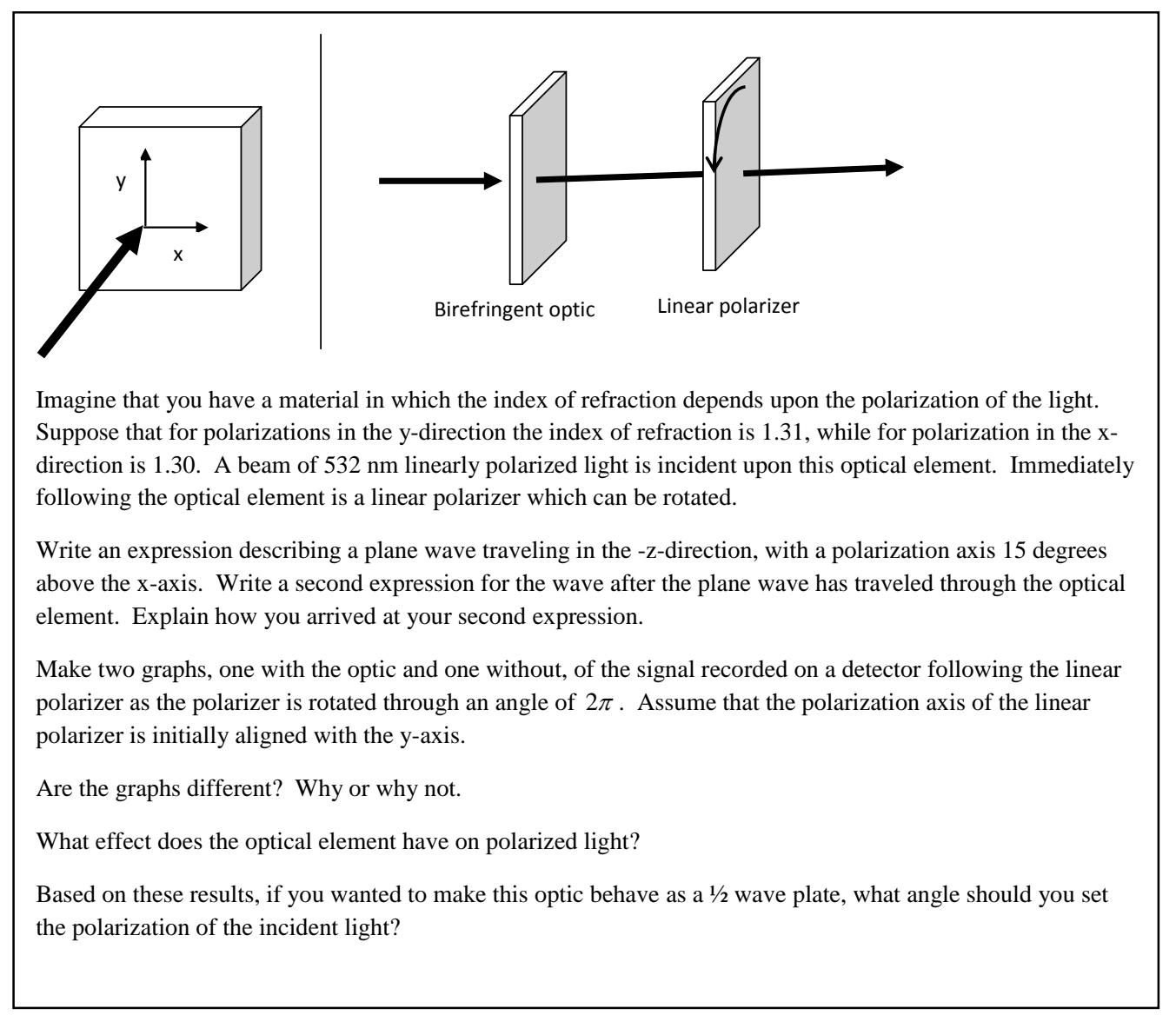

Figure 6. Application tutorial for using polarization

\section{LABORATORY}

In the United States, the laboratory often has goals of "showing" phenomena to students, "familiarizing" students with classic or traditional investigations, to "teach" students about the nature of scientific investigations, to "teach" students about data acquisition and analysis, and/or to "demonstrate" to the students what they have learned in class. With these goals, students can easily become overwhelmed because they are confronted with new apparatus, new phenomena, new physical concepts, and new analysis techniques in almost every laboratory. What is learned in the first laboratory investigation may not apply to laboratory investigation eight! All this newness results in the students exclaiming "Tell me what I need to do, how to do it and what it means!" A request with which all too many instructors are willing to comply by providing detailed directions. This is much as Robert Millikan lamented ${ }^{11}$ that "modern laboratory work in Physics often degenerates into a servile following of directions ...". Fortunately, intermediate optics laboratory typically consists of conceptually limited topics. The problem is how to avoid developing simply the "skill in manipulation" Millikan talks about and assist the students grasp the principles or the concepts.

For intermediate optics students, we set two outcomes: developing laboratory independence, and developing an understanding of optics such that they could design their own optical experiments. Success of achieving each of these goals can be directly measured by providing a task for students in which we provide no guidance; we only observe their work and behavior. Independence can be monitored by observing how they set up an optical system (do they construct a reasonable optical system using appropriate mounts or do they 
resort to sealing wax, string and stacked books), and whether they construct an appropriate optical system to accomplish the task at hand. Student understanding can also be monitored through their discussions.

To be consistent with interactive engagement the laboratory must have two components: students discovering optical physics through experimentation and peer teaching. We want the students to predict and test their predictions, and to confront and articulate their misconceptions. We also want students to develop greater independence in the laboratory to the extent that they can use their new understanding of optics complete a project with virtually no instructor involvement beyond a project goal. This requires that the students be familiar with the equipment as well as our progressively providing briefer instructions since giving too many instructions runs counter to fostering student independence. To achieve these goals we have changed the laboratory to one with a conceptual focus. Students are confronted with materials that elicit their beliefs for a variety of optical situations on which they may have misconceptions. These situations include some that are well documented ${ }^{12-16}$ and others we discovered through our own observations. The students are forced to reflect upon the validity of their beliefs in light of their observations.

To perform a reasonably complex optical experiment, we identified certain basic skills and concepts as listed below. However, not all investigations lend themselves to this approach; we had to select certain key topics (in particular we excluded interferometry as it always seems more demonstration based than experiment based).

\section{Concepts in the laboratory}

- Point and extended sources

- What is an image?

- Virtual and real images

- Image location (are virtual images really there?)

- Does a real image require a screen?

- Point to point correspondence between image and object.

- All rays do not pass through the focal points

- Why use curved mirrors?

- Polarization: what are linear, circular and elliptical polarization

- Understanding linear, $1 / 2$ wave and $1 / 4$ wave polarizers
Skills acquired in the laboratory

- Optics handling skills

- Use of Lenses

- Imaging systems

- Use of mirrors for alignment

- Maintaining polarization through reflection

- Use of polarizers and waveplates

Our academic semester consists of 16 weeks with a 3 hour laboratory activity each week. Every laboratory investigation consists of four parts: a pre-laboratory assignment; class discussion about this assignment, the laboratory activity, and a final task. The pre-laboratory assignment is completed and turned in prior to the laboratory session and serves the following purposes: 1 ) to give the students a chance to think about key topics for the upcoming activity; 2) to provide a topic of discussion for the beginning of the laboratory; and 3) to give us (the instructors) insight into the students' understanding.

The first 11 laboratory investigations consist of conceptual and skill building activities set up so that students must make predictions about certain situations, collect empirical evidence to support or refute these predictions, and reconcile the observations with their predictions. Leading questions within the activity help bring resolution to the activity. Throughout the sequence, later activities are dependent upon the results of earlier activities. The laboratory sequence is listed below. At the end of a laboratory period, the students are required to complete a "final task" in which they are asked to predict the outcome of a new scenario not covered in laboratory, and then test their prediction. 


\section{Laboratory Sequence}

Week 1. Point and extended sources - adapted from Tutorials in Physics

Week 2. Refraction (planar refraction) [virtual images and location]

Week 3. Image formation (curved surface refraction - using beakers) [real and virtual images]

Week 4. Single thin lens - real images and image location using webcam

Week 5. Single thin lens - meaning of focal point

Week $6 . \quad$ Real and virtual images formed with lenses

Week 7. Plane mirrors, images and optical alignment skills

Week 8. Spherical mirrors and image locations

Week 9. Polarization - linear, half-wave plate [ learning to use polarizers and what polarization means]

Week 10. Polarization - quarter-wave plate [is circularly polarized light the same as unpolarized light?]

Week 11. Polarization and reflection [maintaining polarization through reflection]

Week 12-16 - The remaining 5 weeks are spent on the optics project. Projects have been, static Fourier

transform spectrometers, frustrated total internal reflection, optical activity of corn syrup, and the construction of a spectrograph. ${ }^{17}$

The investigations are performed by small groups (three students per group) and the students must come to a group consensus on any answer. These groups are assigned by the faculty and change three times through the semester. Initially, academically weaker students are paired with academically stronger students. The students are informed that they must be able to work independently and cannot rely solely upon their classmates for performing certain tasks. They are individually responsible for their own knowledge/skills. The last groupings pair students with the same academic level. This means that there always is that one group consisting entirely of students who do not seem to have been contributed significantly to their previous groups. We have found that at least one of those students greatly improves in this environment.

\section{Webcam}

Because the laboratory requires the students collect empirical evidence of their observations, in particular for locations of image and orientation of the image, we make extensive use of web cameras. Some of these cameras have the lens removed. These are used either to image directly upon the sensor, or replace the lens with a $50 \mathrm{~mm}$ FL lens (fixed in position relative to the sensor). The $50 \mathrm{~mm}$ FL lens gives the camera a fairly small depth of field and allows it to serve the purpose determining the location of an image - even a virtual image.

\section{Sample activities}

Figure 7 is a laboratory fragment that elicits student ideas on the issue of all of the light rays passing through a focal point. Once they have made predictions, they have to test the situation and see if they were correct. This type of situation provides the students with a "low-stakes" opportunity for failure, which is one of the most critical learning tools.

Once students learn about lenses, they often see no use for those silly concave and convex mirrors. The problem is that students often do not see any reason that they should make use of curved mirrors. In this activity chromatic aberration becomes very apparent as the location of the image, depending upon color changes visibly for the lens, but does not change at all for the mirror. Of course, the mirror introduces aberration due to its use off axis. A laboratory fragment is shown for students to explore curved mirrors is shown in Figure 8, 


\section{Lenses Part II}

This section explores the nature of a focal point in regard to the rays that form an image. This section also explores the effects on an image as a result of forcing all the rays to pass through a small hole.

Set up the light source, $200 \mathrm{~mm}$ focal length lens, and a ruled screen on an optical rail. Adjust the distances between the screen, lens, and source so that a clear image appears on the screen. Place the webcam on the optical rail and get the image to appear on the monitor screen.

Consider what would happen if we add an iris to this set-up and remove the $\mathrm{cm}$ ruled screen. A student suggests that an iris will have a minimal effect if it is placed at the focal point of the $200 \mathrm{~mm}$ lens (see the sketch shown below). The student explains that this works because all the rays must pass through the focal point. Do you agree or disagree with this students reasoning? EXPLAIN YOUR THOUGHTS.

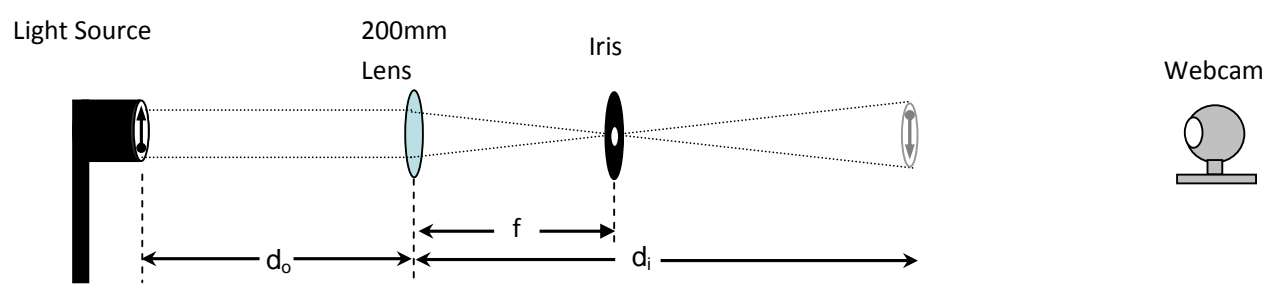

Figure 7 - Fragment of a laboratory having the students reconsider through experimentation their ideas of image formation and ray paths.

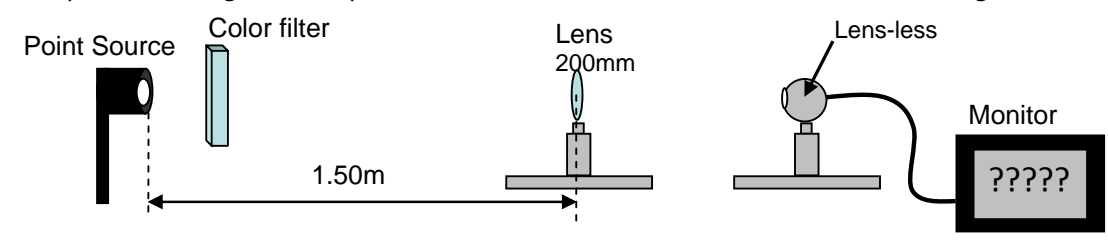

Adjust the lens and record the distance from the lens to the camera for each of the color lenses Make sure you readjust the focus each time. Note: you may have to adjust the shutter speed of the camera so that it doesn't saturate.

Are all of the image distances the same? Should they be? Explain.

Now set up the following using a $200 \mathrm{~mm}$ focal length concave spherical mirror. Try to keep the angle $\phi$ as small as possible. Adjust the mirror, camera distance for each color filter.

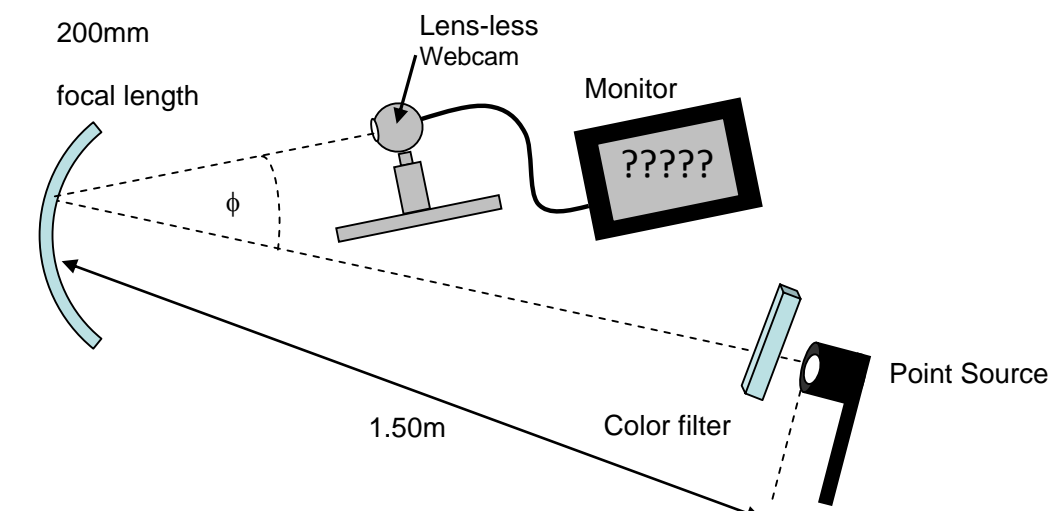

Figure 8 - A laboratory fragment in which the students discover a reason for using curved mirrors rather than lenses. 
Students generally believe the sole test for polarization is rotation of a linear polarizer indicating no preferential polarization angle. Unfortunately, under this criteria, circularly polarized light qualifies as "unpolarized" light. In this investigation, the students explore unpolarized light from a light bulb and then circularly polarized light created using a quarter waveplate. In the former case, the students cannot change the polarization to linear using a quarter waveplate. While in the latter, they are able to do so, making this discovery valuable in persuading them that unpolarized light is not the same as circularly polarized light. In Figure 9, a fragment of this investigation is shown.

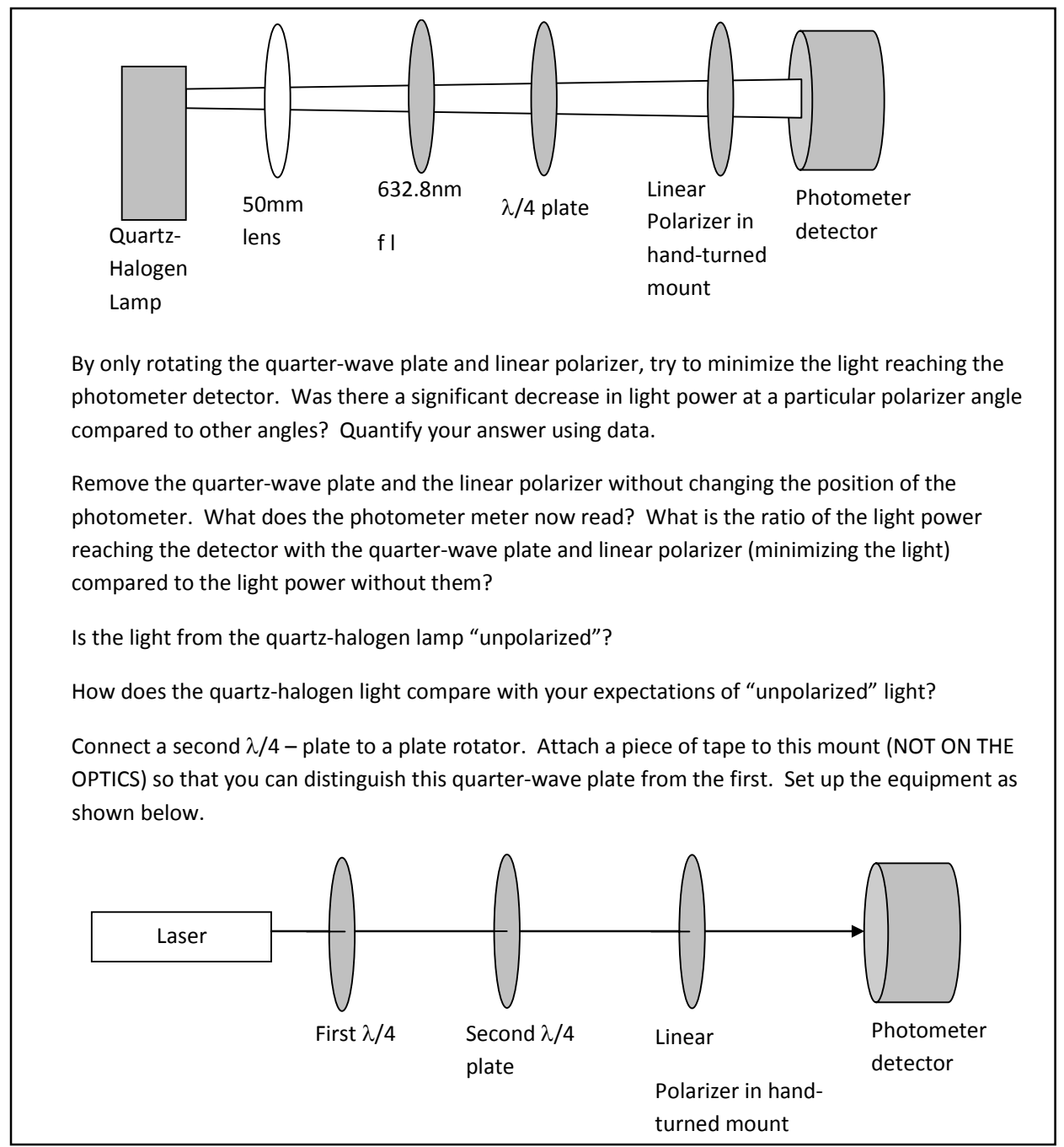

Figure 9 - Laboratory fragment in which the students explore the difference between circularly polarized light and unpolarized light which they often confound.

\section{Laboratory Conclusions}

These laboratories provide a strong background for the students to learn, not only how to use optics, but also how optics work. The eliciting of their thoughts in a low-stakes scenario, and providing the opportunity to acquire empirical evidence with which to refute their predictions enables them to gain a mastery which they might not have been afforded had they simply been following explicit directions. This is the meaning of active learning. 


\section{OVERALL CONCLUSIONS}

These tutorials and laboratories are designed to help the students actively work with the material. However, they cannot be used effectively without instructor to question and lead and they are not intended to be used as such. They are meant to spur discussion between the students, to help the students discover optical phenomena and physics and build an understanding of the same. These tutorials and laboratories are available at (http://users.ipfw.edu/masters/Optics CCLI Project/optics ccli project.htm)

\section{ACKNOWLEDGMENTS}

This work was supported by United States National Science Foundation Grant \# DUE 0410760

\section{REFERENCES}

[1] R. Hake, Interactive-engagement Versus Traditional Methods: A Six-thousand Student Survey of Mechanics Test Data for Introductory Physics Courses, American Journal of Physics 66 64-74 (1998).

[2] E. Mazur, Peer Instruction: A User's Manual, Prentice Hall, Upper Saddle River, 1997.

[3] C. Crouch and E. Mazur, Peer Instruction: Ten years of experience and results, American Journal of Physics 69 970-977 (2001).

[4] D. Sokoloff and R. Thornton, Using Interactive Lecture Demonstrations to Create an Active Learning Environment, The Physics Teacher 35340 (1997).

[5] E. Redish, J. Saul, and R. Steinberg, On the effectiveness of active-engagement microcomputer-based laboratories, American Journal of Physics 65 45-54 (1997).

[6] D. Sokoloff, P. Laws, and R. Thornton, RealTime Physics: active learning labs transforming the introductory laboratory, European Journal of Physics 28 S83-S94 (2007).

[7] R. Hake, Comment on "How do we know if we are doing a good job in physics teaching?", American Journal of Physics 70 1058-1059 (2002).

[8] L. C. McDermot and P. Shaffer, Tutorials in Introductory Physics, Prentice Hall, 2002.

[9] A. V. Heuvelen, Active Learning Problem Sheets, Hayden-McNeil Publishing, 1996.

[10] D. P. Maloney.

[11] R. A. Millikan, Mechanics Molecular Physics and Heat, Ginn, Boston, 1903.

[12] P. Colin and L. Viennot, Using two models in optics: Students' difficulties and suggestions for teaching, American Journal of Physics 69 S36-S44 (2001).

[13] I. Galili, S. Bendall, and F. Goldberg, The effects of prior knowledge and instruction on understanding image formation, The Journal of Research in Science Teaching 30 271-301 (1993).

[14] I. Galili and F. Goldberg, Left-Right Conversions in a Plane Mirror, The Physics Teacher 31463 (1993).

[15] I. Galili, F. Goldberg, and S. Bendall, Some reflections on plane mirrors and images, The Physics Teacher 29 471 (1991).

[16] F. Goldberg and M. L.C., An investigation of student understanding of the real image formed by a converging lens or concave mirror, American Journal of Physics 55 108-119 (1987).

[17] T. T. Grove and M. F. Masters, A student assembled spectrograph with a CCD detector to assist with students' understanding of spectrometry, European Journal of Physics 28 747-753 (2007). 